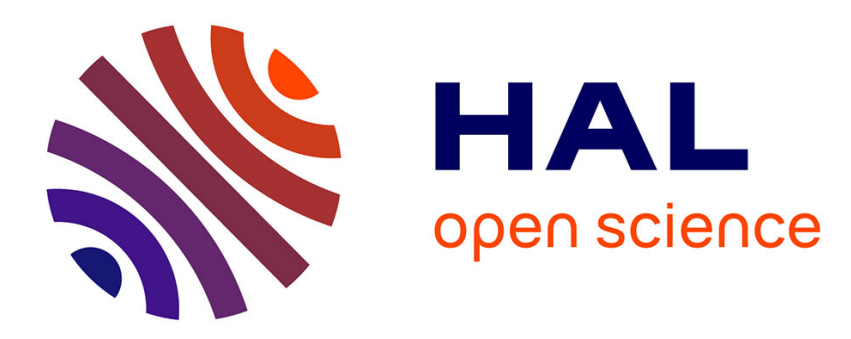

\title{
How to repair a second-order surface for computer experiments by Kriging.
}

Astrid Jourdan

\section{To cite this version:}

Astrid Jourdan. How to repair a second-order surface for computer experiments by Kriging.. 2007. hal-00175059

\section{HAL Id: hal-00175059 \\ https://hal.science/hal-00175059}

Preprint submitted on 26 Sep 2007

HAL is a multi-disciplinary open access archive for the deposit and dissemination of scientific research documents, whether they are published or not. The documents may come from teaching and research institutions in France or abroad, or from public or private research centers.
L'archive ouverte pluridisciplinaire HAL, est destinée au dépôt et à la diffusion de documents scientifiques de niveau recherche, publiés ou non, émanant des établissements d'enseignement et de recherche français ou étrangers, des laboratoires publics ou privés. 


\title{
HOW TO REPAIR A SECOND-ORDER SURFACE FOR COMPUTER EXPERIMENTS BY KRIGING
}

\author{
Astrid JOURDAN \\ LABORATOIRE DE MATHÉMATIQUES APPLIQUÉES DE PAU \\ UMR CNRS 5142 \\ Bâtiment I.P.R.A - Université de Pau et des Pays de l'Adour \\ avenue de l'Université BP 1155 \\ 64013 PAU CEDEX
}

\begin{abstract}
Designs and analysis of computer experiments have been widely investigated in the past decade. Two approaches seem adopted by engineers, the standard response surface methodology and the Kriging method. This paper describes and compares them briefly in order to present a methodology for computer experiments based on these two classical methods. The objective is to combine the advantages of the two approaches, notably the low cost of the standard experimental designs and the accurate response surface of the Kriging method. The use and the efficiency of this tool is demonstrated empirically through examples.
\end{abstract}

KEY WORDS : Computer-Aided Designs, Response Surface, Spatial Statistics, Optimal design.

\section{INTRODUCTION}

For many scientific phenomena, physical experimentation is very expensive, timeconsuming, or impossible. Engineers and scientists have been in the forefront of developing mathematical models and numerical solutions to describe physical systems. As models become more sophisticated, computer run times increase rapidly, and computer experiments are necessary to characterize the physical phenomena.

A computer experiment consists of running a simulation with an input vector $\mathrm{x}$ which specifies the values of some governing input parameters of the computer model. The outputs, $\mathrm{y}\left(\mathrm{x}_{1}\right), \ldots, \mathrm{y}\left(\mathrm{x}_{\mathrm{n}}\right)$, at a given set of inputs $\mathrm{x}_{1}, \ldots, \mathrm{x}_{\mathrm{n}}$, are used to provide a predictor of the simulated response. We are faced with two questions :

- How to select the simulation locations $\mathrm{x}_{1}, \ldots, \mathrm{x}_{\mathrm{n}}$ ?

- Which statistical model is appropriate for the computer responses $\mathrm{y}\left(\mathrm{x}_{1}\right), \ldots, \mathrm{y}\left(\mathrm{x}_{\mathrm{n}}\right) ?$ 
The context and the objectives of computer experiments should be specified before answer the two questions. The context is generally the following.

- The experiments are deterministic, that is re-running the code with the same inputs provides identical outputs.

- Each simulation is time consuming, and thus the number of simulations is limited.

- The computer response is complex since it comes from sophisticated mathematical models, and the complexity is not masked by a random error.

Two kind of objectives are investigated :

- Identify the input parameters influencing the computer response for a better understanding of the physical phenomena,

- Provide a response surface replacement for the computer model for prediction or optimization.

In this paper, we describe and comment briefly on two classical approaches of this problem, the standard experimental design methodology and the Kriging method. The first uses well-known designs, as factorial or composite designs, to fit a polynomial regression model. The second prefers a more sophisticated statistical model taking into account correlation between observations and uses "space-filling" designs to fit it. Our comments highlight the advantages and inconvenient of the two approaches within the context of computer experiments and the two objectives above. Based on that comparison and on our use of the two methods in the petroleum industry, we suggest a sequential approach for computer experiments. This method consists of running an adaptation of the two traditional methods in a sequential way in order to benefit both, the low cost of the standard experimental designs, and the accurate response surface of the Kriging method. The paper is written from a practical point of view, but we refer to many referees throughout the article for more theoretical details. The objective is to provide a tool for computer experiments, efficient in terms of accuracy and simulation cost, and easy to implement. 


\section{STANDARD EXPERIMENTAL DESIGN APPROACH}

\subsection{Description}

A second-order response surface is widely used for computer experiments (see for instance Iman and Helton 1988 [6] or Dejean and Blanc 1999 [5]). This well-known technique consists of using a standard experimental design, such as a Box-Behnken design or a composite design (Box and Draper, 1987 [3], Khuri and Cornell (1996) [10]), to select a set of values of the input parameters $\mathrm{x}_{\mathrm{i}}$. The outputs of the computer code (the observations) are used to fit the second-order polynomial model,

$$
y=\beta_{0}+\sum_{i} \beta_{i} x_{i}+\sum_{i<j} \beta_{i j} x_{i} x_{j}+\sum_{i} \beta_{i} x_{i}^{2}+\varepsilon
$$

where $\varepsilon \sim \mathrm{N}\left(0, \sigma^{2}\right)$ is the error term. Model (1) includes interactions $\mathrm{x}_{\mathrm{i}} \mathrm{x}_{\mathrm{j}}$ and quadratic terms $\mathrm{x}_{\mathrm{i}}{ }^{2}$ according to the design properties.

We note that the main difference between physical experiments and computer experiments is the deterministic output of the computer code. The response is observed without error in computer experiments. From this there follows some adaptations of the standard response surface methodology, notably the experiment designs are used without replication. The error term, $\varepsilon$, is due to the model bias and no longer to the sampling variation. The error term may include a measurement error. Indeed, the object of study is not usually the computer code itself, but the simulated phenomena which can be considered as the computer response plus a random error. This error is due to the simplification of the mathematical models or to the systematic error of the numerical scheme.

Residual plots, variable selection methods and other statistical tools in regression analysis are used for the selection of the best statistical model. The final estimated polynomial model (2) is the response surface which replaces the computer model for prediction or other applications. Let $\mathrm{D}=\left\{\mathrm{x}_{1}, \ldots \mathrm{x}_{\mathrm{n}}\right\}$ be an experimental design. The computer outputs at the design points, $\mathrm{Y}^{\mathrm{T}}=\left[\mathrm{y}\left(\mathrm{x}_{1}\right), \ldots, \mathrm{y}\left(\mathrm{x}_{\mathrm{n}}\right)\right]$, are required for the estimation of the unknown in Model (1),

$$
\hat{y}(x)=X(x) \hat{\beta}
$$

where $\hat{\beta}=\left(\mathrm{X}^{\mathrm{T}} \mathrm{X}\right)^{-1} \mathrm{XY}$ is the least-square estimate of $\beta$ with the design matrix $\mathrm{X}=\left[\mathrm{X}\left(\mathrm{x}_{1}\right), \ldots, \mathrm{X}\left(\mathrm{x}_{\mathrm{n}}\right)\right]^{\mathrm{T}}$ (Khuri and Cornell, $\left.1996[10]\right)$. 
REMARK : Statistical tools such as cross validation are not appropriated for experimental design. Indeed, removing a point destroys the structure of the design, which then is no longer adapted to the polynomial model.

\subsection{Why a second-order surface for computer experiments}

A simple polynomial model can be adequate for modeling the computer response even if the code is very complex. The following examples describe suitable cases.

- The computer response may be very smooth in practice despite the complexity of the code. For instance, in the petroleum industry, the fluid flow simulator provides the cumulative oil production which is usually regular enough to be represented by a second-order surface (Dejean and Blanc, 1999 [5]).

- In a number of applications, the study domain may be smaller than the variation domain of the input parameters. The computer response can be represented by a second-order surface on such a restrictive experimental domain even if the response is not smooth. In the previous example, the input parameters of the fluid flow simulator are known within a range of uncertainty due to measurement error during the phase of characterization of the oil reservoir. The experimental domain represents the uncertainty of the input parameters and is small enough to justify the use of the polynomial model, even for complex responses of the simulator such as water or gas production (Jourdan and Zabalza-Mezghani, 2004 [7]).

- The polynomial model may be useful for performing a sensitivity analysis. Iman and Helton (1988) [6] found in a number of examples that the response surface is inadequate for representing the complex output of the computer code, but could be useful for ranking the importance of the input parameters.

The second-order response surface is widely used by engineers since the methodology is well-known and available in commercial packages, and the interpretation of the polynomial model is easy.

\subsection{Advantages and inconvenience}

The advantages of a second response surface are mainly,

- the simplicity of the methodology, 
- the low cost of simulation.

If a second-order polynomial model is accurate, this approach is the simplest and cheapest method for modeling the computer response. Nevertheless, the statistical model may be too simple when considering the complexity of the simulated phenomena. In a number of cases, the computer response requires a more sophisticated approach such as the Kriging method. Moreover, most of the points in a standard experimental design are located on the edge of the experimental domain and do not allow the detection of irregularities within the domain (Fig. 3a).

\section{STANDARD KRIGING APPROACH}

Recent literature (Sacks et al., 1989 [14,15], Bates et al. 1966 [2], Koehler and Owen, 1996 [11]) suggests that a simple polynomial model may not be appropriate for modeling complex computer processes. For example, second-order response surfaces do not have a very flexible shape. They are incapable of modeling surfaces with multiple extrema. Thus, a spatial model has been adapted from the Kriging model used in geostatistics.

\subsection{Description}

The replacement model adopted in such cases considers the deterministic response as a realization of a random function, $\mathrm{Y}$, that includes a regression model,

$$
\mathrm{Y}(\mathrm{x})=\mathrm{X}(\mathrm{x}) \beta+\Gamma(\mathrm{x})
$$

where $\mathrm{x}=\left(\mathrm{x}_{1}, \ldots, \mathrm{x}_{\mathrm{d}}\right)$ represents the input parameters, $\mathrm{X}(\mathrm{x})$ is a $\mathrm{p}$-vector of regression functions on $\mathbb{R}^{\mathrm{d}}, \beta$ is a p-vector of the unknown parameters of the regression and $\Gamma$ is a Gaussian process with mean zero and covariance function given by

$$
\operatorname{cov}(\Gamma(\mathrm{x}), \Gamma(\mathrm{y}))=\sigma^{2} \mathrm{R}(\mathrm{x}, \mathrm{w})
$$

where $\sigma^{2}$ is the variance and $\mathrm{R}(\mathrm{x}, \mathrm{w})$ is the correlation function depending on a correlation parameter $\theta$,

$$
\mathrm{R}(\mathrm{x}, \mathrm{w})=\exp \left(-\theta \sum_{\mathrm{i}=1}^{\mathrm{d}}\left(\mathrm{x}_{\mathrm{i}}-\mathrm{w}_{\mathrm{i}}\right)^{2}\right), \forall \mathrm{x} \in \mathbb{R}^{\mathrm{d}}, \forall \mathrm{w} \in \mathbb{R}^{\mathrm{d}}
$$

The correlation between observations depends

- on the correlation parameter : the correlation decreases as $\theta$ increases, 
- on the distance between observations : the correlation decreases as the distance increases and two observations are assumed uncorrelated if the distance is large enough.

The correlation parameter defines the distance of correlation in the model.

REMARK : Different correlation functions (exponential, spherical, Matérn, ...) characterizing the computer response smoothness have been investigated in the literature (refer to Chritensen, 1990 [4] or Koeher and Owen, 1996 [11], for an overview of the correlation functions). Moreover, the correlation parameter $\theta$ can take into account different distances of correlation on each axis ( $\theta$ is then a dvector). These solutions may be more adapted than a simple Gaussian process. Note however, that a complex correlation model requires more simulations for the estimation of its parameters.

Let $\mathrm{D}=\left\{\mathrm{x}_{1}, \ldots \mathrm{x}_{\mathrm{n}}\right\}$ be an experimental design. The computer output at the design points, $\mathrm{Y}^{\mathrm{T}}=\left[\mathrm{y}\left(\mathrm{x}_{1}\right), \ldots, \mathrm{y}\left(\mathrm{x}_{\mathrm{n}}\right)\right]$, are required for the estimation of the unknown parameters $\beta, \sigma^{2}$ and $\theta$ in Model (2). Once, $\theta$ is specified. We introduce the notation, $\mathrm{X}=\left[\mathrm{X}\left(\mathrm{x}_{1}\right), \ldots, \mathrm{X}\left(\mathrm{x}_{\mathrm{n}}\right)\right]^{\mathrm{T}}$ as the design matrix, $\mathrm{R}=\left(\mathrm{R}\left(\mathrm{x}_{\mathrm{i}}, \mathrm{x}_{\mathrm{j}}\right)\right)_{\mathrm{i}, \mathrm{j}=1, \ldots, \mathrm{n}}$, is the matrix of correlation between the design points, $r(x)=\left[R\left(x_{1}, x\right), \ldots, R\left(x_{n}, x\right)\right]^{T}$ the vector of correlation between $\mathrm{x}$ and the design points. The best linear unbiased predictor (BLUP) is given by (Sacks et al., 1989 [15], Christensen, 1990 [4], Koehler and Owen, 1996 [11])

$$
\hat{Y}(x)=X(x) \hat{\beta}+r(x)^{T} R^{-1}[Y-X \hat{\beta}]
$$

where $\hat{\beta}=\left(\mathrm{X}^{\mathrm{T}} \mathrm{R}^{-1} \mathrm{X}\right)^{-1} \mathrm{XR} \mathrm{R}^{-1} \mathrm{Y}$ is the generalized least-square estimate of $\beta$. The predictor minimizes the mean square error (MSE),

$$
\operatorname{MSE}(x)=E[Y(x)-\hat{Y}(x)]^{2}=\sigma^{2}\left(1-r(x)^{T} R^{-1} r(x)+K(x)^{T}\left(X^{T} R^{-1} X\right)^{-1} K(x)\right),
$$

where $K(x)=\left[X(x)-r(x)^{T} R^{-1} X\right]^{T}$. The variance $\sigma^{2}$ is estimated by

$$
\hat{\sigma}^{2}=\frac{1}{n}(Y-X \hat{\beta})^{T} R^{-1}(Y-X \hat{\beta}) .
$$

The correlation parameter $\theta$ has to be specified in (3), (4) and (5). Under Gaussian assumptions, $\theta$ could be estimated by maximum likelihood (Mardia and Marshall, 1984 [12]. However, the numerical optimization of the likelihood is time consuming 
and leads usually to a local maximum (Warnes and Ripley, 1987 [16]). In this paper, we select the correlation parameter which minimizes the empirical integrated mean squared error,

$$
\operatorname{IMSE}=\frac{1}{\mathrm{G}} \sum_{\mathrm{k}=1}^{\mathrm{G}} \operatorname{MSE}\left(\mathrm{x}_{\mathrm{k}}\right) / \sigma^{2}
$$

where $\mathrm{x}_{\mathrm{k}}, \mathrm{k}=1, \ldots, \mathrm{G}$ are the $\mathrm{G}$ points of a grid in the experimental domain. The size of the grid $\mathrm{G}$ can be large since (6) does not require the computer response at $\mathrm{x}_{\mathrm{k}}$. Moreover, if $\mathrm{G}$ is large enough, the optimal $\theta$ is independent to the grid size.

\subsection{Kringing as an interpolation model}

In computer experiments there is no measurement error. In this case, the random Gaussian process $\Gamma$ represents the systematic departure from the assumed regression model and leads to an interpolation model, $\hat{Y}\left(x_{i}\right)=Y\left(x_{i}\right)$. The two terms in the right side of (3) are uncorrelated. Adding the second term $r(x)^{T} R^{-1}[Y-X \hat{\beta}]$ to the regression model corrects for the difference between the regression prediction $\mathrm{X}(\mathrm{x}) \hat{\beta}$ and the observation (Fig. 1).

This aspect of the model is interesting for modeling a computer output since the responses are deterministic. Furthermore, the interpolation permits the detection of possible irregularities of the complex computer response.

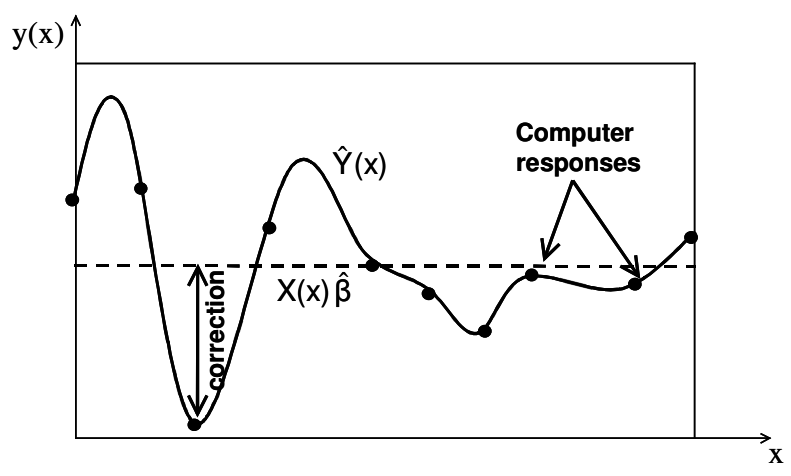

Figure 1. The Kriging response surface behavior in the case of a constant regression

REMARK : Sacks et al., 1989 [15] and Jourdan, 2002 [8] suggest adding an error term in Model (2), called a nugget effect in geostatistics. This term removes the interpolation constraint and seems useful when the interpolation model leads to an irregular response surface. The error term smoothes the surface. In addition, when 
considering physical experiments, the exact interpolation is no longer required and the error term represents a measurement error. The technique presented in this paper in the context of computer experiments can thus be applied to physical experiments.

\subsection{The choice of the regression model}

In a number of examples of the literature (Welch et al., 1992 [17]), the regression model is chosen constant and the regression is the mean of the observations (computer responses). The predictions are then entirely determined by

- the correlation function chosen a priori by the user

- the estimation of the correlation parameter $\theta$ which is not numerically accurate (see previously $\S 3.1$ ).

Figure 2 illustrates that a constant regression is unstable when the correlation parameter $\theta$ varies. A more sophisticated regression, such as polynomial (Sacks et al., 1989 [14]) or trigonometric regression (Bates et al., 1996 [2], Jourdan, 2002 [8]), is required to make the estimated response surface independent of the $\theta$ variations.
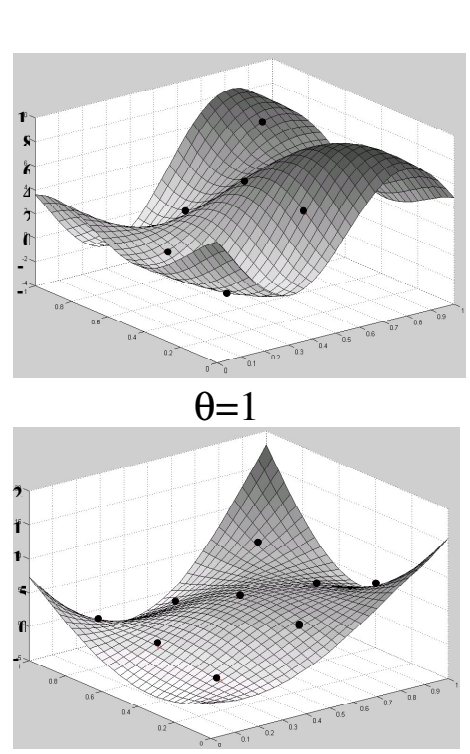

(9)

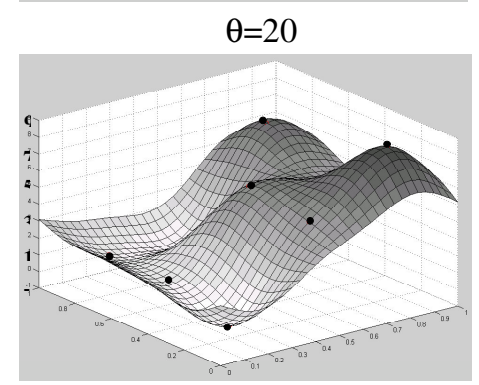

Constant Regression
Trigonometric Regression

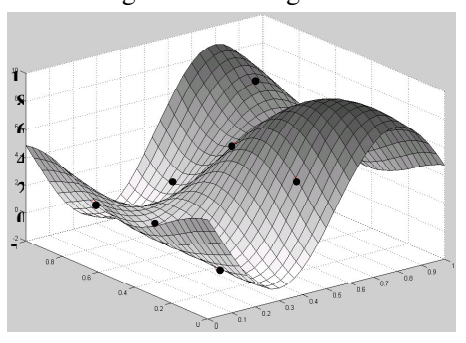

$$
\theta=20
$$

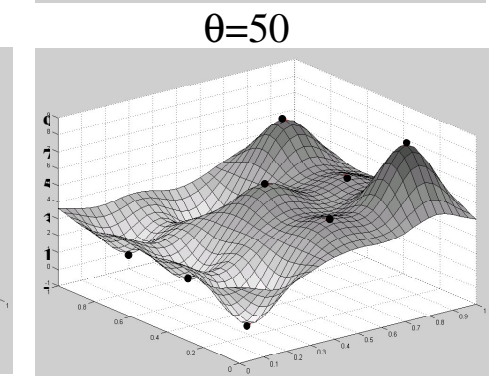

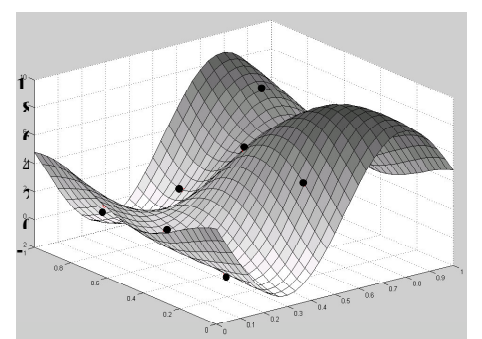

$\theta=50$

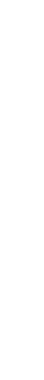

Figure 2. Variation of the Kriging response surface according to the correlation parameter for two kind of regressions

\subsection{Space Filling Designs}


Standard designs (such as Box-Behnken, factorial or composite designs) are not adapted to complex computer codes, and inappropriate for Kriging models. These designs select points essentially on the edge of the experimental domain (Fig. 3a), which is inadequate for

- catching possible irregularities of the computer response within the experimental domain,

- $\quad$ estimating the correlation parameter $\theta$ since only large distances are tested by the design.

Experimental designs used for the Kriging model fit are called 'space filling designs'. They fill up the experimental domain in a uniform fashion and then are suitable for answering the two objectives above. Different kinds of space filling designs have been explored in the bibliography : Latin hypercubes, orthogonal arrays, low discrepancy sequences as one-generator lattices,... (Bates et al., 1996 [2], Koehler and Owen, 1996 [11]).

The more often used designs are Latin hypercubes. Each range of the d input parameters are divided into $n$ equal subintervals and formed a $n \times \ldots \times n$ grid on the experimental domain. A Latin hypercube selects $n$ points among the $n^{d}$ points of the grid such that the $n$ levels of each parameter are represented once in the design (Fig. 3b). Latin hypercubes have the following advantages.

- The construction is easy. Each column of a Latin hypercube is a permutation of $\{1, \ldots, n\}$ or any set of $n$ symbols.

- The design points are uniformly distributed on each axis (the $n$ levels of each input parameter are tested by the design).

Moreover, given $\mathrm{n}$ and $\mathrm{d},(\mathrm{n} !)^{\mathrm{d}}$ Latin hypercubes are possible. The final Latin hypercube can be selected according to a standard Kriging criteria such as maximum entropy, minimum mean squared error or maximin distance (see Park, 1994 [13] for an algorithm and Bates et al., 1996 [2] or Koehler and Owen, 1996 [11] for an overview of the criteria) 


$$
\left(\begin{array}{cc}
0 & 0 \\
1 & 0 \\
0 & 1 \\
1 & 1 \\
0 & 0 \\
\alpha & 0 \\
-\alpha & 0 \\
0 & \alpha \\
0 & -\alpha
\end{array}\right)
$$

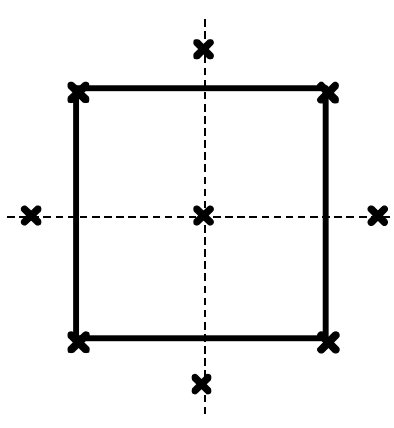

Fig. 3a Composite design

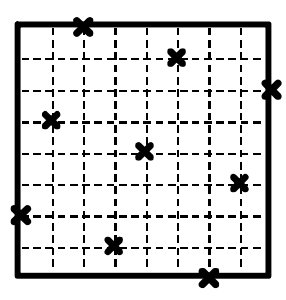

Fig.3b Latin hypercube

$\left(\begin{array}{ll}1 & 3 \\ 2 & 6 \\ 3 & 9 \\ 4 & 2 \\ 5 & 5 \\ 6 & 8 \\ 7 & 1 \\ 8 & 4 \\ 9 & 7\end{array}\right)$

Figure 3. Two nine-point designs for two factors : (a) standard experimental design (b) space-filling design

\subsection{Advantages and inconvenience}

The Kriging appraoch appears more appropriate for computer experiments. The interpolation model has a very flexible shape and is capable of modeling complex surfaces.

On the other hand, this sophisticated model requires more simulations to estimate the unknown parameters, especially for an accurate estimation of the correlation parameter $\theta$. For Latin hypercube, the design size is very flexible and becomes an additional parameter or the problem.

We should also note that the Kriging approach is not adequate for ranking the importance of the input parameters.

\section{SEQUENTIAL APPROACH OF KRIGING}

The standard experimental designs are inexpensive in terms of simulations but can provide a polynomial model which is inappropriate for computer experiments. On the other hand, the Kriging approach provides an efficient model but requires more simulations. The difficulty lies in the selection of the best method (quality/simulation-cost) when no information about the computer response behavior is available. In this instance, we suggest using the two methods in a sequential approach. The objective is to progressively increase the number of simulations in order to improved the response surface, if and only if, the surface requires improvement.

The first step is devoted to the fit of a cheap and simple polynomial model. If the second-order response surface is accurate, the process is stopped. A quality surface 
is obtained for the replacement of the computer program by using a small number of simulations. This step first requires an experimental design for the polynomial model fit. The choice of this design is the first difficulty of the sequential approach since the design should

- possess the good properties of standard response surface designs (see Box and Draper, 1987 [3])

- allow the detection of possible irregularities inside the experimental domain.

If the second-order surface is inaccurate, a second step consists of improving the initial polynomial model by Kriging. The initial design has insufficient number of simulations, and additional points are necessary to fit the new model. The second difficulty of the sequential approach is the location of the new simulations, since adding points must

- provide information where the first-step model is inaccurate in the experimental domain

- test small distances in order to estimate the correlation parameter $\theta$.

In the following sections, we describe in details the two steps with the aid of the following example (Fig. 8.a). We consider the function $f$ as the response of a computer code with two inputs parameters : $\mathrm{x}_{1}$ varying within $[0,2]$ and $\mathrm{x}_{2}$ varying within $[-0.8,3.5]$,

$$
\begin{aligned}
\mathrm{y}=\mathrm{f}\left(\mathrm{x}_{1}, \mathrm{x}_{2}\right) & =2-5 \mathrm{x}_{1}+1.5 \mathrm{x}_{2}+7 \mathrm{x}_{1}{ }^{2}+8\left(1-\mathrm{x}_{1}\right)^{2} \exp \left(-\mathrm{x}_{1}{ }^{2}-\left(\mathrm{x}_{2}+1\right)^{2}\right) \\
& -15\left(\mathrm{x}_{1}-5 \mathrm{x}_{1}{ }^{3}-5 \mathrm{x}_{2}{ }^{4}\right) \exp \left(-\mathrm{x}_{1}{ }^{2}-\mathrm{x}_{2}{ }^{2}\right)-7 \exp \left(\left(\mathrm{x}_{1}+1\right)^{2}-\mathrm{x}_{2}{ }^{2}\right) / 3
\end{aligned}
$$

\subsection{Step1 : Selection of the initial experimental design}

The first step entails fitting a polynomial model at the lowest simulation cost. This step may be extended by Kriging in order to improve the quality response surface. This possibility implies that traditional experimental designs cannot be used due to their point distribution $(\S 2.3)$. On the other hand, the space-filling designs used in the Kriging approach do not have the necessary properties for fitting a polynomial model. In addition, we need a good quality second-order design, especially if the process is stopped after the first step. The suitable designs for a sequential approach 
must have a good spatial point distribution and properties required for a secondorder surface simultaneously.

Kenny, 1998 [9] has developed a class of designs called orthogonal column Latin hypercubes (Table 1) having the two qualities required for our sequential approach.

First, the design contains some of the interesting properties of the standard secondorder surface designs. In particular, the estimates of linear effects of all input parameters are uncorrelated with each other, with the estimates of bilinear interactions and quadratic effects. This property assures a good quality design in terms of D-optimality. Furthermore, the design is adapted for sensitivity studies. The orthogonality makes readable the ranking of the importance of the input parameters since the main effects are independent of interactions or quadratic effects.

Second, as Latin hypercube, the design has a good point distribution. Moreover, one can select the best space-filling design within the class of orthogonal column Latin hypercubes according to standard Kriging criteria.

\begin{tabular}{ccc}
$\mathrm{n}^{\circ}$ & $\mathrm{x}_{1}$ & $\mathrm{x}_{2}$ \\
\cline { 2 - 3 } 1 & 1 & 3 \\
2 & 2 & -4 \\
3 & 3 & -1 \\
4 & 4 & 2 \\
5 & 0 & 0 \\
6 & -4 & -2 \\
7 & -3 & 1 \\
8 & -2 & 4 \\
9 & -1 & -3 \\
\hline
\end{tabular}

Table 1. A $9 \times 2$ orthogonal Latin hypercube with entries from $\{-4,-3, \ldots, 3,4\}$

In our example, we select the orthogonal Latin hypercube detailed in Table 1 and illustrated in Figure $3 b$. The outputs of function $f$ at the design points are used to fit and select a second-order polynomial regression. The accuracy of the estimated model is unsatisfactory since the coefficient of determination $\mathrm{R}^{2}$ is 0.76 and the adjusted coefficient is 0.52 . Figure 4 illustrates the non-influence of the terms in the regression, with exception of the constant. This result is not surprising since the computer surface in Figure 8.a can obviously not be represented by a polynomial surface. The Kriging model seems necessary in this example. 


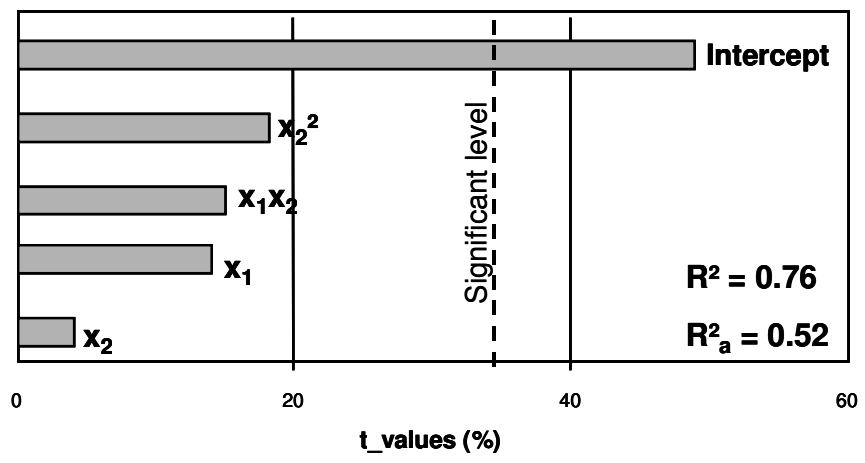

Figure 4. Pareto plot of the selected polynomial regression

\subsection{Step 2 : additional points for Kriging}

The second step consists of improving the model obtained in the first step by adding a Gaussian process to the polynomial model selected previously. The estimation of the unknown parameters of the Gaussian process requires additional simulations. The location of the new design points must respect two objectives.

First, the new points have to test small distances in order to estimate the correlation distance. Note that the smallest distance involved by a Latin hypercube is $\sqrt{\mathrm{d}} / \mathrm{n}$, and provides no information about the correlation between simulations less than this distance.

Second, the new points have to provide local information in the areas of the experimental domain where the polynomial surface is inefficient.

Classical statistical tools are used to detect the points of the initial design which provide an inaccurate prediction (Figure 5). An area is defined around these points and the additional points are randomly chosen in this area, as illustrated in Figure 6. The area is a cube with side length $2 / \mathrm{n}$, centered around the selected point. This cube assures that critical zones are tested as well as distances smaller than $\sqrt{\mathrm{d}} / \mathrm{n}$.

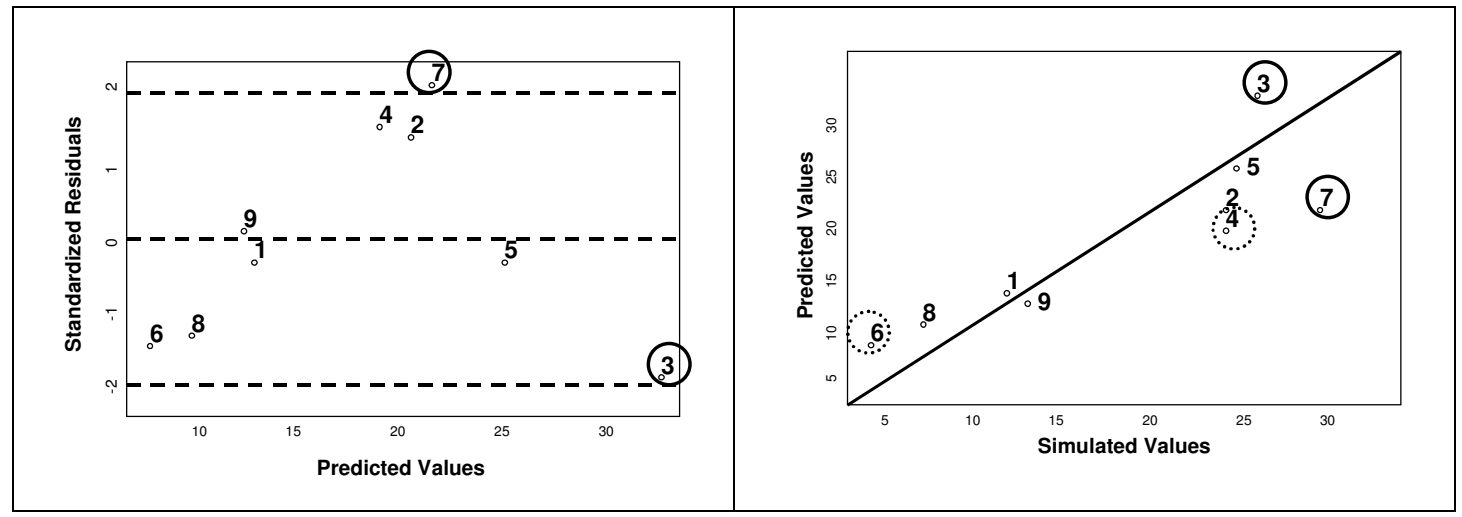


Figure 5. Residual plot and predicted values against simulated (true) values at the design points.

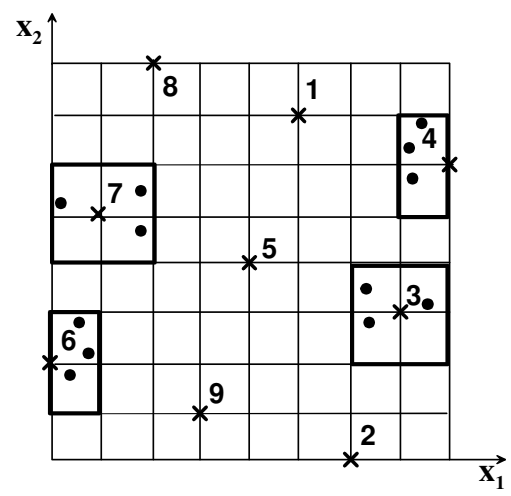

Figure 6. The Latin hypercube of Table 1 (crosses) plus the new simulations (points)

The outputs of the new simulations plus the first-step outputs are used to estimate the correlation parameter $\theta$ by optimizing the IMSE (6). In our example, the optimal $\theta$ equals 0.56 .

REMARK : In this example the inefficient points of the initial design are obviously numbers 7 and 3 (numeration of Table 1), but we can also consider points 4 and 6 . If we add new points only around 7 and 3, the IMSE optimizing process converges on large values of $\theta$, and the matrix of correlation $\mathrm{R}$ becomes numerically unstable. The optimizing process requires additional simulations, and new points around 4 and 6 are necessary. Adding sequentially the new points in the second step assures running a minimum number of simulations.

The Kriging predictor (3) obtained with the polynomial regression and the optimal $\theta$ is used to generate the final response surface (Fig. 8c).

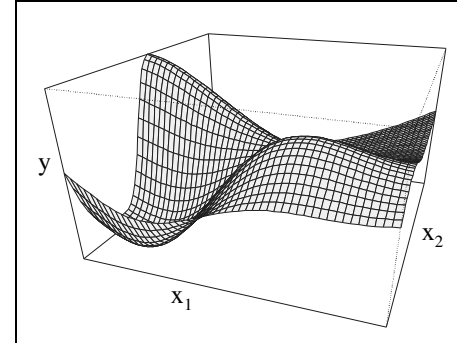

Fig. 8a. Computer surface (function $f$ )

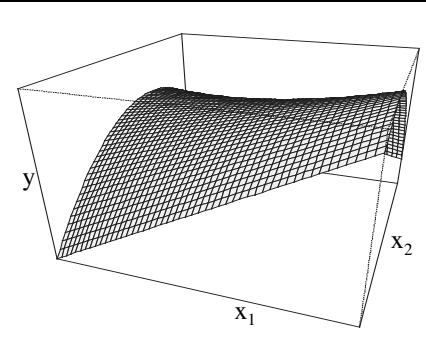

Fig. 8b. Polynomial surface (step 1)

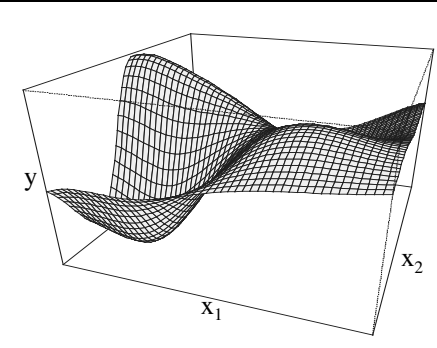

Fig. 8c. Kriging surface (step 2)

Figure 8. Comparison of the two response surfaces 


\subsection{Comparison with standard approaches}

In this example, the final estimated model necessitates 21 simulations, 9 points in the initial design (Table 1) plus 12 additional points (Figure 6).

\section{Comparison with the standard experimental design approach}

In order to compare the two methods, the standard experimental design approach has been applied to the example using composite design requiring 9 simulations. The sequential approach provides either a polynomial surface (Fig. 8b, step 1) or a Kriging surface if the polynomial surface needs to be improved (Fig. 8c, step 2). If the computer response behaves like a polynomial surface, the two methods lead to a satisfactory second-order surface using the same number of simulations. In the example, the initial composite design with two factors in the first step requires 9 simulations. If the computer response is more complex, the standard approach is very poor since the second-order surface cannot be improved. Thus the sequential approach either provides the same result for the same cost, or gives a better quality surface.

\section{Comparison with the standard Kriging approach}

In order to compare the two methods, the standard Kriging approach has been applied to the example using the same model, a second-order polynomial regression plus a Gaussian process, and the same number of simulations, an optimal Latin hypercube of size 21 (maximin distance criteria).

The same quality surfaces have been obtained with the two approaches. The advantage of the sequential approach is that is less expensive in the case of a smooth computer response requiring only a polynomial model. The standard Kriging method has also been applied with smaller Latin hypercubes. The accuracy of the response surface decreases naturally with the number of simulations. The difficulty with this approach is selecting a priori the number of simulations necessary for a quality surface. This problem does not exist with the sequential approach since the design is constructed step by step.

An other example 
We consider the function $g$ (Balkin and Lin, 2000 [1]) as the response of a computer program with two inputs parameters : $x_{1}$ varying within $[0,2]$ and $x_{2}$ varying within $[-0.8,3.5]$

$$
\mathrm{y}=\mathrm{g}\left(\mathrm{x}_{1}, \mathrm{x}_{2}\right)=\left(10 \mathrm{x}_{1}^{4}-20 \mathrm{x}_{2} \mathrm{x}_{1}^{2}+10 \mathrm{x}_{2}^{2}+\mathrm{x}_{1}^{2}-2 \mathrm{x}_{1}+5\right)^{-1}
$$

The initial design used in the first step is the Latin hypercube of Table 1. The accuracy of the second-order surface is unsatisfactory since the coefficient of determination $\mathrm{R}^{2}$ is 0.62 and the adjusted coefficient is 0.16 . Figure $9 \mathrm{~b}$ illustrates the poor performance of the polynomial surface. Hence, a Kriging model is fitted by adding new simulations around points 5, 6, 7 and 9. The Kriging surface (Figure 9c) is less accurate than in the previous example but is obviously better than the polynomial surface (Figure 9b), especially when searching for the maximum value. The true surface maximum is 0.25 and occurs at $x_{1}=1.0$ and $x_{2}=1.0$. The maximum provided by the Kriging surface is 0.26 and occurs at $\mathrm{x}_{1}=1.15$ and $\mathrm{x}_{2}=0.88$, whereas the polynomial surface gives a maximum of 0.16 found at the extremities of the experimental domain. We note that Balkin and Lin, 2000 [1] obtained the quite same quality result with a neural network approach.

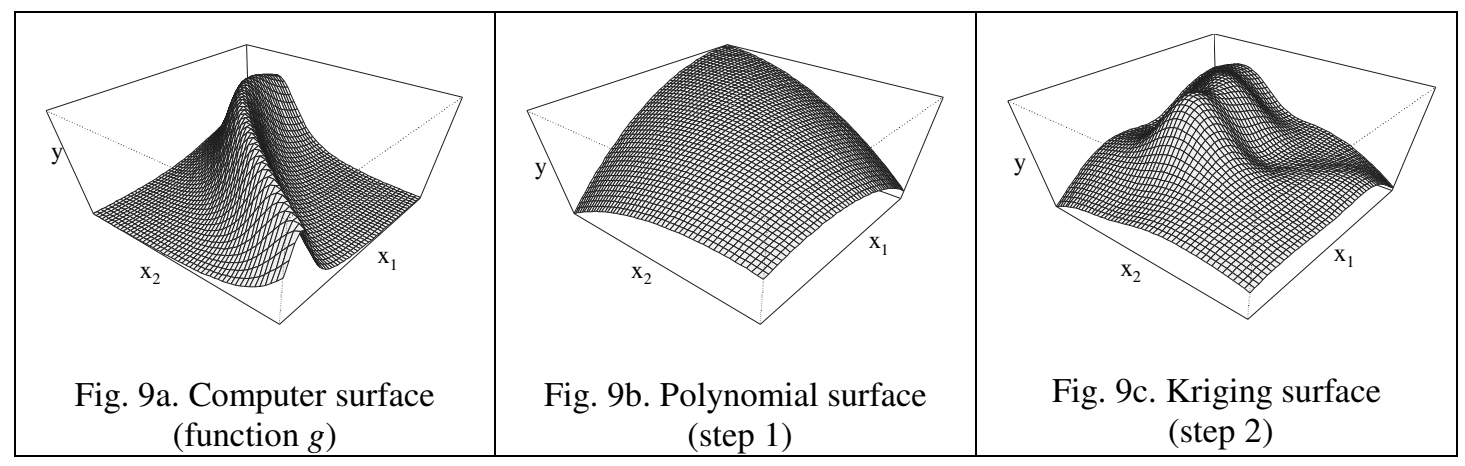

Figure 9. Comparison of the two response surfaces

\section{DISCUSSION}

The interest of the approach presented in this paper is to adapt two standard methods in order to run them sequentially. The two objective are achieved since the method improves the poor performance of a second-order response surface and reduces the number of simulations required by Kriging (in the case of a smooth computer response). This approach can be considered as repairing a second-order surface using the computer experiments already run. 
The paper shows initial work on this method and many questions are to develop, especially concerning the Kriging step (definition of the area, number of new simulations in each area, quantify the information provided by the new experiments...). The sequential Kriging approach appears promising and will be applied to a reservoir oil production simulation problem.

\section{REFERENCES}

[1] Balkin, S.D.; Lin, D.K.J. A Neural Network Approach to Response Surface Methodology. Commun. Statist. - Theory Meth., 2000, 29, 2215-2227.

[2] Bates, R.A.; Buck, R.J.; Riccomagno, E.; Wynn, H.P. Experimental Design and Observation for large Systems. J. R. Statist. Soc. B, 1996, 58, 77-94.

[3] Box, G.E.P.; Draper, N.R.. Empirical Model-Building and Response Surfaces, New York : John Wiley, 1987.

[4] Christensen, R. Linear Models for Multivariate, time series, and spatial Data, SpringerVerlag, 1990.

[5] Dejean, J.-P.; Blanc, G.; Managing Uncertainties on Production Prediction Using Integrated Statistical Methods. Paper SPE 56696 presented at the 1999 SPE Annual Technical Conference and Exhibition, Houston, U.S.A., 3-6 October 1999, 1999.

[6] Iman, R.; Helton, J.C. An Investigation of Uncertainty and Sensitivity Analysis Techniques for Computer Models. Risk Analysis, 1988, 8, 71-90.

[7] Jourdan, A.; Zabalza-Mezghani, I. Response surface designs for scenario management and uncertainty quantification in production. Mathematical Geology, 2004 (to appear).

[8] Jourdan, A. Approches Statistiques des Expériences Simulées. Rev. Statistique Appliquée, 2002, $L, 49-64$.

[9] Kenny, Q.Y. Orthogonal Column Latin Hypercubes and their Applications in Computer Experiments. J. of American Statistical Association, 1998, 93, 1430-1439.

[10] Khuri, A. I.; Cornell, J. A. Response surfaces : designs and analyses (Second Edition). Statistics : textbooks and monographs, volume 152, Marcel Dekker, 1996.

[11] Koehler, J.R.; Owen, A.B. Computer Experiments. In Ghosh, S., Rao, C.R., (Eds.), Handbook of Statistics, 13 : Designs and Analysis of Experiments, North- Holland, Amsterdam, 1996, 261-308.

[12] Mardia, K.V.; Marshall, R.J. Maximum Likelihood Estimation of Models for Residual Covariance in Spatial Regression. Biometrika, 1984, 71, 135-146.

[13]Park, J.S. Optimal Latin Hypercube Designs for Computer Experiments. J. of Statist Planning and Inference, 1994, 39, 95-111. 
[14] Sacks, J; Schiller, S.B.; Welch, W.J. Designs for Computer Experiments. Technometrics, 1989, 31, 41-47.

[15] Sacks, J.; Welch, W.J.; Mitchell, T.J.; Wynn, H.P. Design and analysis of Computer Experiments. Statistical Science, 1989, 4, 409-435.

[16] Warnes, J.J.; Ripley, B.D. Problem with Likelihood Estimation of Covariance Functions of Spatial Gaussian Processes. Biometrika, 1987, 74, 640-642.

[17] Welch, W.J.; Buck, R.J.; Sacks, J.; Wynn, H.P.; Mitchell, T.J.; Morris, M.D. Screening, Predicting and Computer Experiments. Technometrics, 1992, 34, 15-25. 